\title{
Resonant coupling and negative differential resistance in metal/ferrocenyl alkanethiolate/STM structures
}

\author{
Shuchun Wang, Wenchang Lu, Qingzhong Zhao, and J. Bernholc \\ Center for High Performance Simulation and Department of Physics, North Carolina State University, Raleigh, \\ North Carolina 27695-7518, USA \\ (Received 24 August 2006; published 22 November 2006)
}

\begin{abstract}
Recent experimental studies demonstrated that self-assembled molecules sandwiched between metallic contacts can perform logic functions based on negative differential resistance (NDR). To understand the mechanism of NDR, the electronic structure and transport properties of one such junction, ferrocenyl alkanethiolate attached to a gold surface and probed with a scanning tunneling microscope tip, are investigated by large scale $a b$ initio calculations. The $I-V$ characteristics show strong NDR features at both positive and negative biases, in good agreement with the experimental data. The voltage-dependent transmission, potential drop profile, and molecular level alignment under bias suggest that the ferrocenyl group acts like a quantum dot and that the NDR features are due to resonant coupling between the highest occupied molecular orbital and the density of states of gold leads. The strength of the individual NDR peaks can be tuned by changing the tunneling distance or using suitable spacer layers.
\end{abstract}

DOI: 10.1103/PhysRevB.74.195430

\section{INTRODUCTION}

Ever since the concept of using individual molecules as functional electronic devices was first proposed in the $1970 \mathrm{~s},{ }^{1}$ having individual molecules perform the basic functions of conventional electronic components became the goal of molecular electronics. Recently, this field has experienced dramatic growth, due to the emerging need for alternate routes toward smaller, faster, and cheaper integrated circuits. Key electronic components, such as wires, diodes, and transistors, have been successfully demonstrated, all based on single or self-assembled molecules sandwiched between electrodes. For self-assembled molecules on a single-crystal surface, the substrate serves as one of the electrodes. A variety of methods are used to form the contact with the other electrode, such as a scanning tunneling microscope (STM) tip $^{2-6}$ and atomic-scale break junctions. ${ }^{7,8}$ A number of novel and promising characteristics for these types of molecular devices have been reported. For example, negative differential resistance (NDR) (Refs. 3 and 9) and molecular memory effects $^{10,11}$ have been demonstrated on a system of phenylethylene oligomers functionalized with different side groups. Recently, a ferrocene-based molecular wire with nearly perfect conductance was reported by Getty et al. ${ }^{12}$ The ballistic conductance thus reaches the criteria for real applications of molecular electronic devices. NDR has also been observed for ferrocenyl-undecanethiolate self-assembled monolayers $\left(\mathrm{Fc}-\mathrm{C}_{11} \mathrm{~S}-\mathrm{SAM}\right)$ on $\mathrm{Au}(111)$ surface. ${ }^{13,14}$

Self-assembled molecular systems with NDR are promising devices, due to their utility in fast switching logic and simplicity in integration. However, in many cases the NDR mechanisms have not been unraveled either from experiments or from theory, hampering the development of molecular devices. In many cases, theoretical understanding of the electron transport properties and of the underlying NDR mechanisms is critical for the design of such devices. For ferrocenyl-alkanethiolate SAM on $\mathrm{Au}(111)$ surface, the redox effect, i.e., a change of the charge state with bias, has been suggested as an explanation of the NDR. ${ }^{13}$ However,
PACS number(s): 73.63.-b, 72.10.-d, 73.50.Fq

resonant tunneling may also play an important role. Another possibility is some level of bond breaking when a large bias is applied, ${ }^{15}$ resulting in a change of the atomic structure of the molecule or a change at the molecule-substrate interface.

In this paper, we study the electronic and transport properties of ferrocenyl-pentanethiolate SAM on $\mathrm{Au}(111)$. The atomic structures of $\mathrm{C}_{5} \mathrm{~S}$ and $\mathrm{Fc}-\mathrm{C}_{5} \mathrm{~S}$ on the $\mathrm{Au}(111)$ surface and their saturation coverages are determined by total-energy calculations. Our nonequilibrium quantum transport calculations for geometry-optimized structures show NDR features at both negative and positive biases, in good agreement with the experimental data. ${ }^{13}$ The mechanism responsible for the NDR is uncovered by analyzing the molecular level alignment, transmission spectra under bias, and charge transfer between the molecule and the STM tip.

\section{CALCULATIONS}

The calculations use a massively parallel real-space multigrid implementation ${ }^{16}$ of density-functional theory (DFT) ${ }^{17}$ Due to the relatively large sizes of the supercells, the $k$-space sampling is restricted to the $\Gamma$ point. The exchange and correlation terms are represented by the generalized gradient approximation (GGA) in Perdew, Burke, and Ernzerhof form. ${ }^{18}$ The electron-ion interactions are described by nonlocal, ultrasoft pseudopotentials. ${ }^{19}$ A partial core correction is added to the $\mathrm{Au}$ and $\mathrm{Fe}$ pseudopotentials in order to account for the nonlinearity in the exchange-correlation term. ${ }^{20}$ The wave functions and localized orbitals are described on a grid with spacing of $0.20 \AA$. A double grid technique ${ }^{21}$ is employed to evaluate the inner products between the nonlocal potentials and the wave functions, thereby substantially reducing the computational cost and memory without losing accuracy.

\section{RESULTS AND DISCUSSION}

\section{A. Atomic structure}

First, the equilibrium structures of the adsorbed molecules on the $\mathrm{Au}(111)$ surface are investigated by total-energy cal- 
(a)

(b)

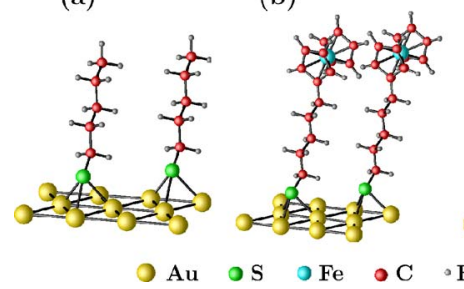

(c)

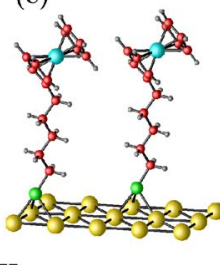

${ }^{\circ} \mathrm{H}$

FIG. 1. (Color online) Atomic structures of (a) $\mathrm{C}_{5} \mathrm{~S}-\mathrm{Au}(111)$ $(\sqrt{3} \times \sqrt{3}) R 30^{\circ}$, (b) $\quad \mathrm{Fc}-\mathrm{C}_{5} \mathrm{~S}-\mathrm{Au}(111) \quad(\sqrt{3} \times \sqrt{3}) R 30^{\circ}$, and (c) $\mathrm{Fc}-\mathrm{C}_{5} \mathrm{~S}-\mathrm{Au}(111)(\sqrt{21} \times \sqrt{7})$.

culations. The gold substrate is simulated by a six-layer slab. For each of the structures, the molecule is anchored with a sulfur atom to the $\mathrm{Au}(111)$ surface and all the atoms are relaxed using first-principles forces. For the adsorption of alkanethiolate on the $\mathrm{Au}(111)$ surface, two structural models have been reported. One is a $(\sqrt{3} \times \sqrt{3}) R 30^{\circ}$ reconstruction with low molecular coverage. ${ }^{22}$ The other is a $c(4 \times 2)$ reconstruction with high molecular coverage. ${ }^{23}$ Since the molecular density for the $c(4 \times 2)$ structure is too high when ferrocenyl heads are present, we only consider the $(\sqrt{3} \times \sqrt{3}) R 30^{\circ}$ structure, see Fig. 1(a). Our calculations show that the fcc hollow site is favorable and that the molecular axes are tilted by $27^{\circ}$ with respect to the surface normal. These results are in agreement with recent theoretical work $^{24,25}$ and experiments. ${ }^{22}$

In the case of $\mathrm{Fc}_{-} \mathrm{C}_{5} \mathrm{~S}$ on $\mathrm{Au}(111)$, our calculations for the $(\sqrt{3} \times \sqrt{3}) R 30^{\circ}$ Fc superlattice [Fig. 1(b)] show that the repulsive interaction between the neighboring ferrocenyls is very strong, resulting in a positive adsorption energy. To determine the saturation coverage, we calculated adsorption energies for a number of different structures and coverages. Among the structures that were considered, two molecules per $(\sqrt{21} \times \sqrt{7})$ unit cell is the maximum coverage for which the adsorption energy is still negative $(-0.3 \mathrm{eV}$ per molecule in this case). For this coverage, the optimized tilt angle of the molecular axis is $15^{\circ}$. Clearly, the ferrocenyl head greatly decreases the molecular packing density on the $\mathrm{Au}(111)$ substrate.

\section{B. Electron transport}

The nonlinear $I-V$ characteristics are evaluated using the nonequilibrium Green's function (NEGF) methodology ${ }^{26,27}$ in a basis of optimal localized orbitals. ${ }^{28,29}$ The atomcentered orbitals are optimized variationally in the equilibrium geometry. Four orbitals for hydrogen, six for carbon, eight for sulfur, and ten for iron and gold atoms are used, all with the radius of $4.50 \AA$. The Hartree potential is obtained by solving the Poisson equation with boundary conditions matching the electrostatic potentials of the left and right gold leads, respectively. The surface Green functions are calculated with a transfer-matrix technique in an iterative scheme. ${ }^{30} \mathrm{~A}$ self-consistent calculation is carried out for each bias, which is critical in order to obtain correct $I-V$ characteristics at large biases. ${ }^{31}$ Due to the efficiency of our $O(N)$
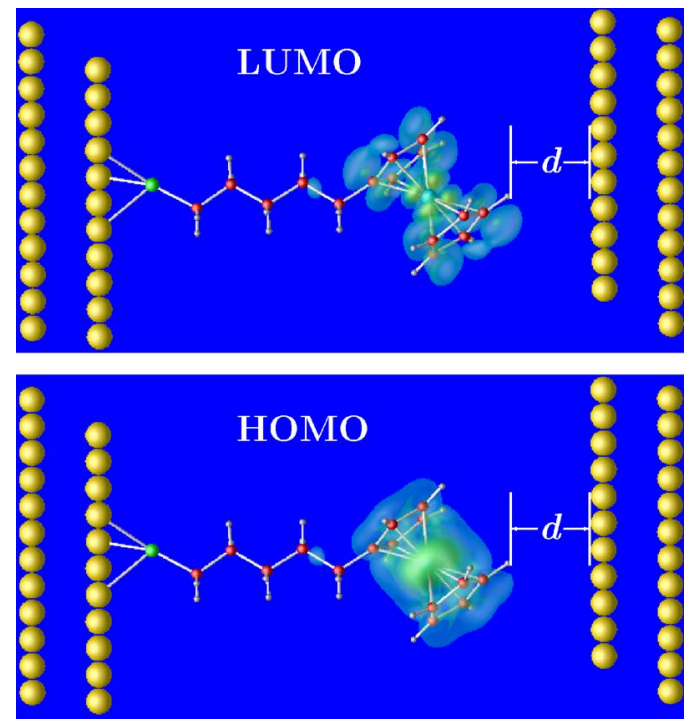

FIG. 2. (Color online) Schematic views of the $\mathrm{Au}-\mathrm{SC}_{5}-\mathrm{Fc}-\mathrm{Au}$ system, where $d$ is the distance between the molecule and the STM tip, and of isosurfaces of the HOMO and LUMO orbitals at zero bias. Both the HOMO and the LUMO are localized on the ferrocenyl head.

methodology, ${ }^{28,29}$ we are able to include 372 atoms and a total of nearly 4000 electrons in the calculations.

Figure 2 shows a schematic view of the system, together with the highest occupied molecular orbital (HOMO) and the lowest unoccupied molecular orbital (LUMO). In our NEGF calculations, the conductor part includes eight layers of $\mathrm{Au}$ on the left, the molecule, and seven layers of $\mathrm{Au}$ on the right. As described above, the ferrocenyl pentanethiolate is anchored with a sulfur atom to the $\mathrm{Au}(111)$ surface (left part in Fig. 2). For simplicity, we model the STM tip as another $\mathrm{Au}(111)$ surface (right part in Fig. 2) in the calculations. The effects of the shape of the STM tip will be investigated in the future. In experiments, the distance $d$ between the molecule and the STM tip is determined by the setpoint current. The lower the current, the larger the distance, since the magnitude of the tunneling current depends exponentially on $d$.

The transmission in the molecular junction is mainly determined by molecular orbital(s) with energy levels near the Fermi level of the metal leads in contact with the molecule. These orbitals are usually either the HOMO or the LUMO. Their isosurfaces are plotted in Fig. 2. Clearly, both the HOMO and the LUMO are localized on the ferrocenyl head.

After the potential profile is self-consistently determined, the transmission spectrum under the external applied bias $V$ can be calculated by

$$
T(E, V)=\frac{2 e^{2}}{h} \operatorname{Tr}\left[\Gamma_{L}(E) G_{C}^{+}(E) a_{R}(E) G_{C}^{-}(E)\right]
$$

where $G_{C}^{ \pm}$are the advanced and retarded Green functions of the conductor, and $\Gamma_{L, R}$ are the coupling functions to the left and right leads, respectively.

The calculated transmission spectra $T(E, V)$ are shown in Fig. 3, with the Fermi level of the gold substrate at zero bias 


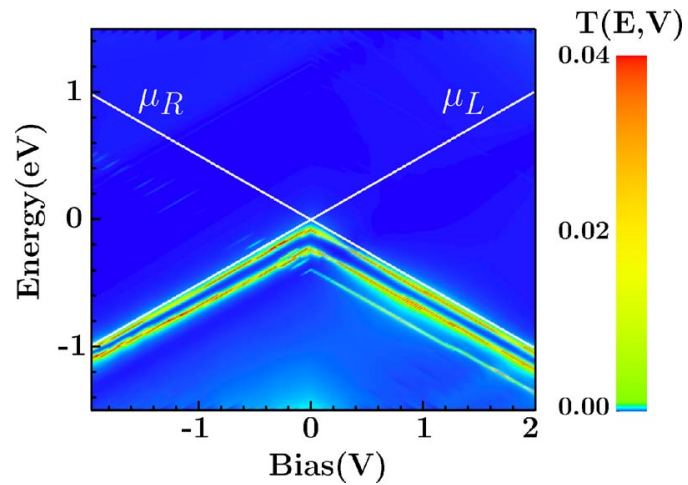

FIG. 3. (Color online) Calculated transmission spectra $T(E, V)$ for the $\mathrm{Au}(111)-\mathrm{SC}_{5}-\mathrm{Fc}-\mathrm{Au}(111)$ junction with $d=3.39 \AA$. The two white lines are the chemical potentials of the two leads, $\mu_{L}=E_{F}+e V / 2$ and $\mu_{R}=E_{F}-e V / 2$, as functions of the applied bias voltage.

chosen as the energy zero. The two white lines show the chemical potentials of the left and right leads, with $\mu_{L}-\mu_{R}$ $=V$ being the effective applied bias. We note that the transmission peak from the HOMO resonance is always near the lower chemical potential for both positive and negative biases. This is because the bias-induced potential drop across the molecular junction is very different for positive and negative biases. At a negative bias, the largest potential drop occurs across the vacuum between the molecule and the right electrode (STM tip), see Fig. 4(b). This indicates that the electrons on the left side of the junction (the Au substrate)

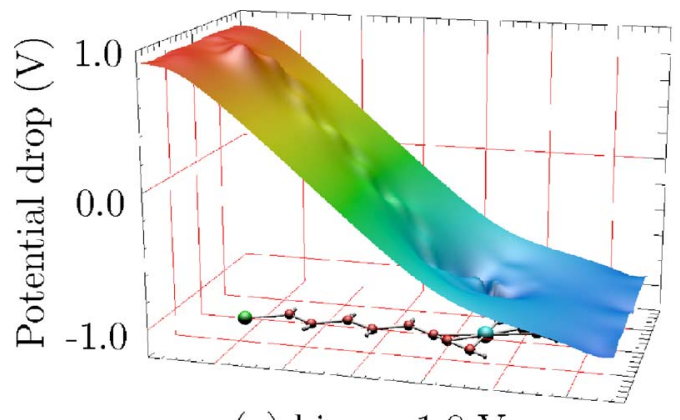

(a) bias $=1.8 \mathrm{~V}$

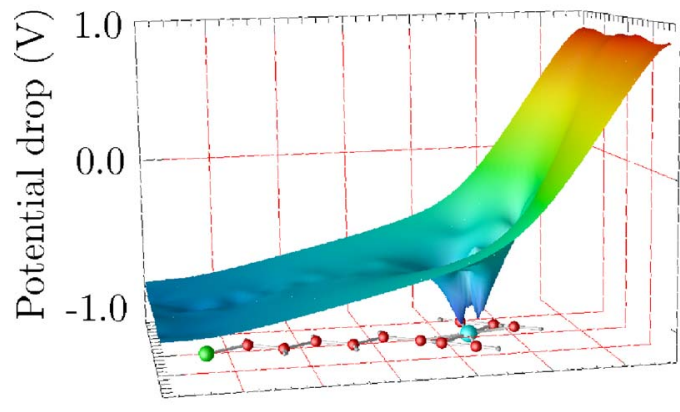

(b) bias $=-1.8 \mathrm{~V}$

FIG. 4. (Color online) Potential drop along the molecule for (a) bias $=1.8 \mathrm{~V}$ and (b) bias $=-1.8 \mathrm{~V}$.

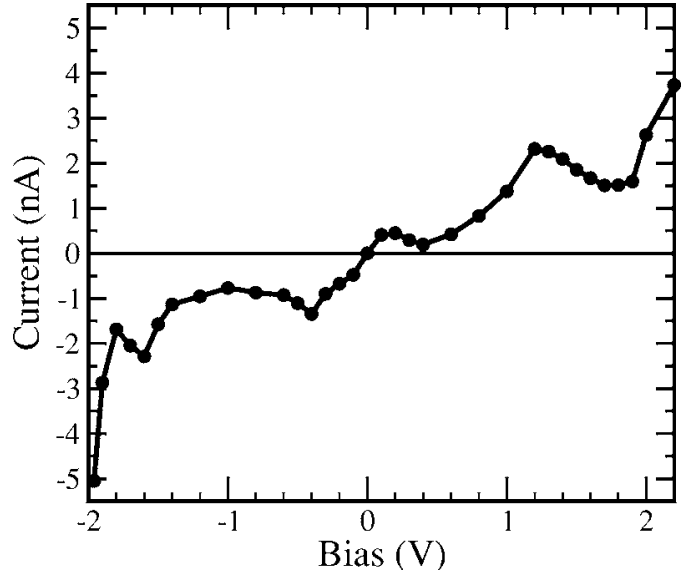

FIG. 5. Current-voltage characteristics of the $\mathrm{Au}(111)-\mathrm{SC}_{5^{-}}$ $\mathrm{Fc}-\mathrm{Au}(111)$ junction with $d=3.39 \AA$.

can move into the molecule to effectively screen the applied field. However, at a positive bias, we find that the potential drops mainly in the carbon chain region of the molecule, as shown in Fig. 4(a). As a result, the average potential around the ferrocenyl head is always aligned with the lower chemical potential. The HOMO, which is localized on the ferrocenyl, is thus pinned by the lower chemical potential.

The current through the molecular junction is given by

$$
I(V)=\int_{-\infty}^{\infty} T(E, V)\left[n_{F}\left(E-\mu_{L}\right)-n_{F}\left(E-\mu_{R}\right)\right] d E,
$$

where $n_{F}$ is the Fermi-Dirac distribution and $T(E, V)$ is the transmission coefficient at bias $V$.

The $I-V$ curve calculated with the STM-molecule distance of $d=3.39 \AA$ is shown in Fig. 5. The NDR features are at $-1.6,-0.4,0.2$, and $1.2 \mathrm{~V}$, compared with the experimentally observed NDR at -1.6 and $1.6 \mathrm{~V}$. Tunneling through the ferrocenyl head is responsible for these features, since the calculated $I-V$ curve without the ferrocenyl, i.e., for pentanethiolate on $\mathrm{Au}(111)$, does not show NDR behavior. This is in good agreement with experiments. ${ }^{13,14}$

All of the NDR features can be understood in terms of resonant tunneling between the two top occupied levels and the gold density of states. In the density of states (DOS) of bulk gold, the first peak below the Fermi energy is at around $-0.2 \mathrm{eV}$ and the second one is at $-1.2 \mathrm{eV}$. When the HOMO level is aligned with one of these peaks, the current reaches its maximum and NDR appears. Figure 6 illustrates the NDR mechanism at $1.2 \mathrm{~V}$ by plotting the DOS of the gold leads, the average potential, and the energy positions of the top two occupied orbitals. These orbitals are localized on the ferrocenyl by potential barriers. One barrier is near the Aumolecule interface, while the other is in the vacuum region between the ferrocenyl and the STM tip. Therefore the ferrocenyl acts like a quantum dot. As shown in Fig. 6, at the bias of $1.2 \mathrm{~V}$ the HOMO level is aligned with the peak in the DOS of left lead. As a result, a current maximum appears. For negative bias, the NDR can be understood in a similar way, except that the resonant coupling is between the HOMO 


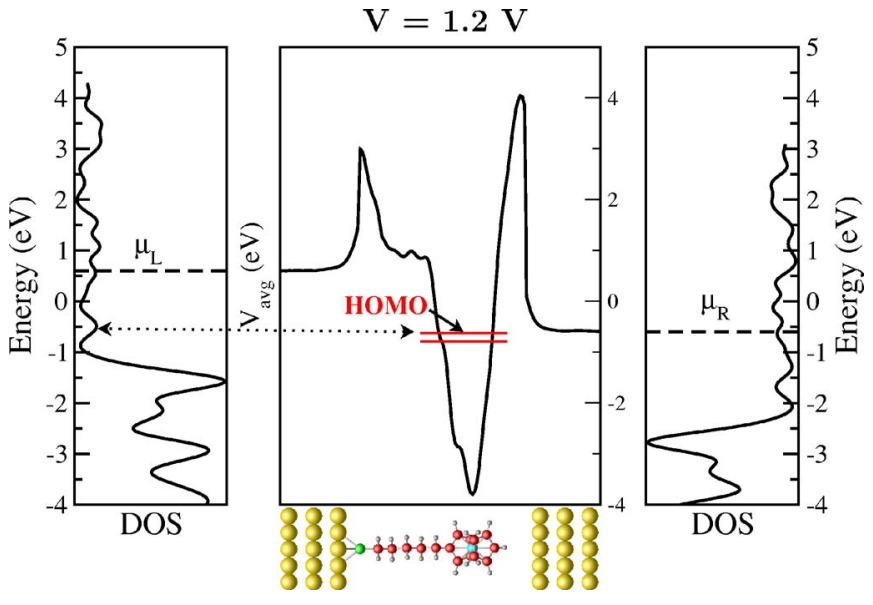

FIG. 6. (Color online) The NDR mechanism for the $\mathrm{Au}(111)$ $\mathrm{SC}_{5}-\mathrm{Fc}-\mathrm{Au}(111)$ junction at $1.2 \mathrm{~V}$. The left and right panels are densities of states (DOS) of bulk gold. The horizontal dashed lines in the DOS plot mark the left and right chemical potentials. The middle panel shows the average potential in the molecular junction. The horizontal solid lines in that panel mark the energies of the HOMO and the level just below the HOMO. These orbitals dominate the transmission.

and the right lead. Charge transfer between the molecule and the right lead also plays an important role in determining the position of the NDR. At a negative bias, charge transfer leads to an internal electric field that cancels out part of the applied external bias. As a result, the NDR appears at a higher voltage for negative bias than for the positive one, because there is no charge transfer for positive bias.

The calculated value of the current in Fig. 5 is about 50 times bigger than that of the STM measurement. This is mainly due to the unknown distance $d$ between the STM tip and the molecule. In order to investigate the effect of this distance on electron transport, we carried out calculations for different values of $d$.

Figure 7 shows the $I-V$ curves for positive bias at dis-

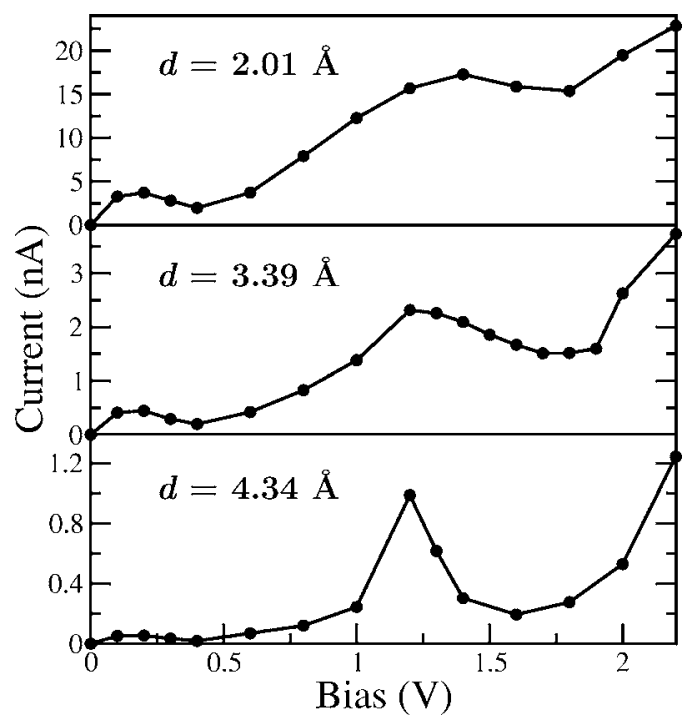

FIG. 7. Current-voltage characteristics of the $\mathrm{Au}(111)-\mathrm{SC}_{5}-\mathrm{Fc}-$ $\mathrm{Au}(111)$ junction as a function of the STM-molecule distance. tances of $2.01,3.39$, and $4.34 \AA$, respectively. Their overall shapes are similar and the NDR positions are at about same biases. However, the absolute value of the current decreases exponentially with an increase of the distance. For example, at the bias of $0.6 \mathrm{~V}$, we can fit the current by $I(d)=I_{0} e^{-\beta d}$ with the decay constant $\beta=0.90$. The distance also affects the shape of the NDR region, or its "strength." Specifically, the $\mathrm{NDR}$ at $1.2 \mathrm{~V}$ is enhanced with increasing $d$. This indicates that a low current setpoint in the STM experiment is important for the observation of NDR at large biases. However, an increase of the distance has the opposite effect on NDR at low bias; the peak at $0.2 \mathrm{~V}$ is attenuated with increasing $d$. This result can explain why the low bias NDR is not seen in the STM experiment, because a high current setpoint would be needed for its observation. This "NDR tuning" effect can be utilized in the design of molecular devices, for example, by introducing spacer layers, which would adjust the strength of the NDR and the working current for optimal device performance.

Obviously, the length of the alkane chain plays a major role in determining the magnitude of the current. For computational reasons, our calculations were performed on ferrocenyl pentanethiolate, while the experiment used ferrocenyl undecanethiolate. However, we performed a few calculations for ferrocenyl undecanethiolate, from which we learned that a current of the same magnitude as in the experiment would be obtained at STM distance of $3.01 \AA$. Nevertheless, the shape and the major features of the distance-dependent $I$ - $V$ curves remain similar to those of ferrocenyl pentanethiolate.

Apart from the distance and length effects, the unknown molecule-gold contact geometry and chemistry could partially explain the remaining discrepancies between theory and experiment. For example, if the molecule is attached to an atomic protrusion rather than an atomically flat surface, the magnitude of the current would be substantially lower. ${ }^{32,33}$ If the STM tip has a locally protruding atom or group of atoms, the transmission would also be affected. For positive bias, the position of the NDR should not vary with the shape of the STM tip, since it is due to a feature in the density of states of the Au substrate. For the negative bias, however, its position may change if the STM tip assumes a different shape, since the local density of states would be altered. In addition, other variables, such as temperature or local disorder near the contact can alter the value of the current measured in experiment.

\section{SUMMARY}

In summary, we investigated theoretically the geometry, electronic structure, and quantum transport properties of ferrocenyl-alkanethiolate self-assembled monolayers on $\mathrm{Au}(111)$ by large-scale first-principles calculations. The calculated NDR features at large biases are in good agreement with experiment. We find that the HOMO is always localized at the ferrocenyl head. It is pinned to the lowest chemical potential of the electrodes. Resonant tunneling through the top two occupied orbitals dominates the $I-V$ characteristics, 
with NDR effects being due to features in the density of states of the gold surface. The magnitude of the current and of the strength of the NDR are dramatically affected by the distance between the molecule and the STM tip. In practical devices, spacer layers will have a similar effect and can be used to tune the performance of the device.

\section{ACKNOWLEDGMENTS}

We would like to thank C. B. Gorman for stimulating discussions. We gratefully acknowledge support by DOD and DOE, and grants of supercomputer time provided by the DOD Challenge Program.
${ }^{1}$ A. Aviram and M. A. Ratner, Chem. Phys. Lett. 29, 277 (1974).

${ }^{2}$ R. M. Metzger, Acc. Chem. Res. 32, 950 (1999).

${ }^{3}$ J. Chen, M. A. Reed, A. M. Rawlett, and J. M. Tour, Science 286, 1550 (1999).

${ }^{4}$ S. Datta, W. Tian, S. Hong, R. Reifenberger, J. I. Henderson, and C. P. Kubiak, Phys. Rev. Lett. 79, 2530 (1997).

${ }^{5}$ Y. Xue, S. Datta, S. Hong, R. Reifenberger, J. I. Henderson, and C. P. Kubiak, Phys. Rev. B 59, R7852 (1999).

${ }^{6}$ S. Hong, R. Reifenberger, W. Tian, S. Datta, J. Henderson, and C. P. Kubiak, Superlattices Microstruct. 28, 289 (2000).

${ }^{7}$ M. A. Reed, C. Zhou, C. J. Muller, T. P. Burgin, and J. M. Tour, Science 278, 252 (1997).

${ }^{8}$ J. G. Kushmerick, D. B. Holt, J. C. Yang, J. Naciri, M. H. Moore, and R. Shashidhar, Phys. Rev. Lett. 89, 086802 (2002).

${ }^{9}$ J. Chen, W. Wang, M. A. Reed, A. M. Rawlett, D. W. Price, and J. M. Tour, Appl. Phys. Lett. 77, 1224 (2000).

${ }^{10}$ J. Chen and M. A. Reed, Chem. Phys. 281, 127 (2002).

${ }^{11}$ M. A. Reed, J. Chen, A. M. Rawlett, D. W. Price, and J. M. Tour, Appl. Phys. Lett. 78, 3735 (2001).

${ }^{12}$ S. A. Getty, C. Engtrakul, L. Wang, R. Liu, S. H. Ke, H. U. Baranger, W. Yang, M. S. Fuhrer, and L. R. Sita, Phys. Rev. B 71, 241401(R) (2005).

${ }^{13}$ C. B. Gorman, R. L. Carroll, and R. R. Fuierer, Langmuir 17, 6923 (2001).

${ }^{14}$ R. A. Wassel, G. M. Credo, R. R. Fuierer, D. L. Feldheim, and C. B. Gorman, J. Am. Chem. Soc. 126, 295 (2004).

${ }^{15}$ J. L. Pitters and R. A. Wolkow, Nano Lett. 6, 390 (2006).

${ }^{16}$ E. L. Briggs, D. J. Sullivan, and J. Bernholc, Phys. Rev. B 54, 14362 (1996)
${ }^{17}$ W. Kohn and L. J. Sham, Phys. Rev. 140, A1133 (1965).

${ }^{18}$ J. P. Perdew, K. Burke, and M. Ernzerhof, Phys. Rev. Lett. 77, 3865 (1996).

${ }^{19}$ D. Vanderbilt, Phys. Rev. B 41, 7892 (1990).

${ }^{20}$ S. G. Louie, S. Froyen, and M. L. Cohen, Phys. Rev. B 26, 1738 (1982).

${ }^{21}$ T. Ono and K. Hirose, Phys. Rev. Lett. 82, 5016 (1999).

${ }^{22}$ F. T. Arce, M. E. Vela, R. C. Salvarezza, and A. J. Arvia, Langmuir 14, 7203 (1998).

${ }^{23}$ J. J. Gerdy and W. A. Goodard, J. Am. Chem. Soc. 118, 3233 (1996).

${ }^{24}$ Y. Yourdshahyan, H. K. Zhang, and A. M. Rappe, Phys. Rev. B 63, 081405(R) (2001).

${ }^{25}$ H. Gronbeck, A. Curioni, and W. Andreoni, J. Am. Chem. Soc. 122, 3839 (2000).

${ }^{26}$ B. Larade, J. Taylor, H. Mehrez, and H. Guo, Phys. Rev. B 64, 075420 (2001)

${ }^{27}$ M. Brandbyge, J. L. Mozos, P. Ordejon, J. Taylor, and K. Stokbro, Phys. Rev. B 65, 165401 (2002).

${ }^{28}$ J. L. Fattebert and J. Bernholc, Phys. Rev. B 62, 1713 (2000).

${ }^{29}$ M. B. Nardelli, J. L. Fattebert, and J. Bernholc, Phys. Rev. B 64, 245423 (2001).

${ }^{30}$ M. P. L. Sancho, J. M. L. Sancho, and J. Rubio, J. Phys. F: Met. Phys. 14, 1205 (1985).

${ }^{31}$ W. Lu, V. Meunier, and J. Bernholc, Phys. Rev. Lett. 95, 206805 (2005).

${ }^{32}$ M. DiVentra, S. T. Pantelides, and N. D. Lang, Phys. Rev. Lett. 84, 979 (2000).

${ }^{33}$ Y. Xue and M. A. Ratner, Phys. Rev. B 68, 115407 (2003). 